\title{
¿Es la exportación un elemento relevante para explicar el rendimiento de una empresa? El caso de las pymes gallegas para el período 2002 - 2013
}

Is export a relevant element to explain the performance of a company? The case of Galician SMEs for the period 2002-2013

José Pablo Abeal Vázquez

Profesor Contratado Interino. Doctor en Economía por la UDC. Departamento de Empresa. Universidade da Coruña. A Coruña. España.

jpabeal@udc.es

Begoña Álvarez García

Profesora Contratada Doctor. Doctora en Economía por la UDC. Departamento de Empresa. Universidade da Coruña. A Coruña. España.

balvarez@udc.es

Javier Tarrío Saavedra

Profesor Contratado Interino. Doctor en Estadística por la UDC. Departamento de Matemáticas. Universidade da Coruña. A Coruña. España.

javier.tarrio@udc.es

Recepción: 24/03/2017. Aceptación: 02/04/2017. Publicación: 24/05/2018

Citación sugerida:

Abeal Vázquez, J.P., Álvarez García, B. y Tarrío Saavedra, J. (2018). ¿Es la exportación un elemento relevante para explicar el rendimiento de una empresa? El caso de las pymes gallegas para el período 2002-2013. 3C Empresa: investigación y pensamiento crítico, 7(2), 10-25. DOI: http://dx.doi. org/10.17993/3cemp.2018.070234.10-25/ 


\section{RESUMEN}

El principal objetivo de este trabajo es averiguar en qué medida el hecho de que una empresa sea exportadora tiene consecuencias sobre su rendimiento. El caso de estudio, elaborado a partir de la base de datos ARDAN, se encuadra dentro de la región española de Galicia. El período temporal comprende desde el año 2002 hasta 2013. La muestra recoge únicamente PYMEs. El análisis empírico parte de un conjunto de variables empresariales ligadas a la gestión, a factores fijos como la localización, y a la coyuntura económica. Los resultados obtenidos con las técnicas de análisis aplicadas no son concluyentes y queda el camino abierto a nuevas investigaciones.

\section{ABSTRACT}

The main objective of this work is to find out to what extent the fact that a company is an exporter has consequences on its performance. The case study, developed from ARDAN database, is focused on the Spanish region of Galicia. The temporary period covers from 2002 to 2013. The sample includes only SMEs. The empirical analysis starts from a set of business variables linked to management, fixed factors such as location and to the economic situation. The results obtained with the analytical techniques applied are not conclusive and the way is open to new investigations.

\section{PALABRAS CLAVE}

Rendimiento empresarial, Características de las empresas, PYMES, Exportación, Análisis multivariante.

\section{KEY WORDS}

Firm performance, Firm characteristics, SME's, Exporting, Multivariate analysis. 


\section{INTRODUCCIÓN}

El binomio entre la actuación de la empresa y su enfoque internacional es una cuestión ampliamente abordada dentro de la investigación económica y desde perspectivas muy variadas durante las últimas décadas (Li, 2007). Sin embargo, se trata de un tema de gran complejidad donde las conclusiones distan de ser unánimes y los interrogantes siguen abiertos y sin perspectivas de una posición común al respecto (Glaum y Oesterle, 2007; Kamakura, Ramón-Jerónimo y Vecino, 2012; Majochi, Dalla y D’Angelo, 2015). En este sentido, las soluciones se asimilan a cada caso de estudio y a unas características concretas.

Las teorías que han servido como soporte a los diferentes autores para sus análisis son muy heterogéneas. Sin embargo, podemos destacar que gran parte de la literatura consideran la internacionalización como un proceso evolutivo (Johanson y Vahlne, 1977), donde las empresas aumentan gradualmente su interés por las operaciones internacionales al considerarlas un factor impulsor de su crecimiento (Kamakura et al., 2012).

Por otra parte, el criterio de proximidad geográfica o cultural suele considerarse una de las claves a la hora de señalar los primeros destinos de exportación. A medida que las empresas afianzan su conocimiento de los mercados internacionales, estas van dando el salto hacia métodos más directos de exportación. No obstante, a partir de la última década del siglo pasado, adquirió fuerza un enfoque que intentaba explicar el hecho, cada vez más relevante, de aquellas empresas que nacían globales o que se transformaban en globales con rapidez (Oviatt y McDougall, 1994).

\section{A medida que las empresas afianzan su conocimiento de los mercados} internacionales, estas van dando el salto hacia métodos más directos de exportación.

El objetivo básico de este trabajo es contrastar, para las pymes gallegas en el período 2002-2013, el papel que juega la exportación para explicar su rendimiento. Para ello, se integra la variable exportación dentro de un modelo que incluye un conjunto de variables internas de cada empresa y de su entorno. El análisis propuesto es exploratorio. A partir de un análisis descriptivo inicial, se plantea un análisis de la varianza y un modelo explicativo mediante una regresión lineal múltiple. A continuación, se plantean brevemente las conclusiones y algunas de las posibles futuras líneas de investigación a desarrollar. 


\section{ESTADO DEL ARTE}

La variedad de teorías, metodologías, datos y conclusiones en la literatura demuestran la falta de consenso en torno a la relación existente entre el rendimiento de las empresas y su capacidad para exportar. Así, algunos autores encontraron evidencia empírica que demostraba la relación positiva entre la actividad exportadora y el rendimiento empresarial en base a una mayor fortaleza competitiva (e. g. Bernard y Jensen, 1999; Ling-Yee, 2004; Annavarjula y Beldona, 2005; Pangarkar, 2008; Ellis, Davies y Wong, 2011).

Otros autores, en cambio, concluyen que, justificado por el principio económico del rendimiento marginal decreciente, la relación puede tener forma de U invertida (Gomes y Ramaswamy, 1999; Li y Qian, 2005). También tenemos aproximaciones que nos hablan de una forma de S, mostrando un proceso de convergencia, deterioro, reorientación y vuelta a la convergencia (Contractor, Kundu y Hsu, 2003; Lu y Beamish, 2004). Incluso, algunos estudios, no encuentran una relación significativa (Ramírez-Aleson y Espitia-Escuer, 2001).

Desde otra perspectiva, Bernard y Jensen (1999) encontraron evidencia empírica de que la actividad de exportar no es causa del incremento de la productividad, pero genera oportunidades de expansión para las empresas con mayor productividad, lo que se traduce en un empleo más estable. A su vez, Yi y Wang (2012) y Serti y Tomasi (2014), demuestran que las variables tamaño y productividad están positivamente relacionadas con la probabilidad de exportar y que la localización y la cercanía a otros exportadores también son dos elementos que contribuyen a reducir costes y lanzarse a exportar.

Desde la perspectiva de las pequeñas y medianas empresas, y para el caso de Italia, Giovannetti, Ricchiuti y Velucchi (2013) demuestran que existe una relación evidente entre el contexto socioeconómico (localización geográfica, cercanía a las infraestructuras, etc.) y su rendimiento y la propensión a exportar. Sin embargo, esta relación es mucho menor en el caso de las grandes empresas.

Otro ejemplo que ilustra la variedad en las conclusiones es el trabajo de Serra, Pointon y Abdou (2012). En él analizan las empresas del sector textil de Reino Unido y Portugal y observan resultados distintos en ambos países: por ejemplo, el tamaño de las empresas es importante en el caso de Portugal, pero no en Reino Unido. 
A la heterogeneidad comentada anteriormente, hay que añadir que abundan los estudios en la literatura que realizan un análisis muy profundo pero a partir de un pequeño número de empresas durante un período de tiempo no muy largo (e. g. Pangarkar, 2008; Majochi et al., 2015; Boehe, Qian y Peng, 2016). Esta aproximación, según indican Kamakura et al. (2012), no siempre capta la naturaleza dinámica de las exportaciones.

Con respecto al tamaño de las empresas analizadas, aunque los trabajos que tienen en cuenta a grandes empresas que cotizan en Bolsa es abundante (Gourlay y Seaton, 2004; Li y Qian, 2005; Annavarjula y Beldona, 2005; Glaum y Oesterle 2007), cada vez son más los autores que enfocan sus estudios hacia las pymes (Westhead, Wright y Ucbasaran, 2001; Kalinic y Forza, 2012; Majochhi et al. 2015).

No todas las variables influyen de igual forma en el proceso exportador. Gourlay y Seaton (2004) observaron que el tamaño de la empresa, la innovación o el capital humano eran atributos esenciales para entrar en los mercados de exportación. En la misma dirección, Kamakura et al. (2012) concluyen que los factores clave para el desarrollo internacional de una empresa son el capital humano, la tecnología y las relaciones gerenciales.

Javalgi, White y Lee (2000) observaron la importancia de incluir características ligadas a la gestión para predecir con mayor precisión el comportamiento exportador. También concluyen que la contribución de las características de cada empresa a la propensión a exportar varía según la industria. En esta línea, Kalinic y Forza (2012) concluyen que el aspecto determinante del éxito en el cambio del proceso de internacionalización de las pymes tradicionales se basa en poseer un enfoque estratégico específico.

Los diferentes trabajos en este ámbito también han tratado la vertiente del apoyo institucional. Así, Westhead, White y Ucbasaran (2004) justificaron que las autoridades de política económica tienen mejores expectativas de éxito animando a las empresas ya exportadoras a redoblar sus esfuerzos en este ámbito, en vez de apoyando el incremento de empresas potencialmente exportadoras. 


\section{METODOLOGÍA}

En esta investigación ${ }^{1}$ se utiliza la base de datos ARDAN. Contiene datos de las empresas de la Comunidad Autónoma de Galicia. Con relación al concepto de pyme, la Comisión Europea discrimina entre pymes y grandes empresas considerando, entre otros criterios, un número de empleados menor de 250. Sin embargo, la cifra de empleados que se suele considerar fuera de Europa es de 500 (Lu y Beamish, 2004; Majochi et al., 2015). Por ello, este estudio ha optado por recoger el conjunto de las empresas gallegas que cuentan con menos de 500 empleados.

El rendimiento o actuación de la empresa se puede recoger a partir de distintas medidas: la cifra de ventas (Gourlay y Seaton, 2004), la ratio de rentabilidad sobre ventas (Contractor et al., 2003, Boehe et al., 2016), la Q de Tobin (Pantzalis, 2001), la rentabilidad económica y la rentabilidad financiera (Lu y Beamish, 2004; Thomas y Eden, 2004; Annavarjula y Beldona, 2005; Majochi et al., 2015). Se ha optado por utilizar la rentabilidad económica debido a la frecuencia de su uso.

Después de realizar un análisis descriptivo básico sobre la exportación en el ámbito geográfico del estudio, se analiza la estructura de dependencia existente entre la variable rentabilidad y diversos factores y variables cuantitativas. Los factores estudiados son el sector de actividad económica, la localización de la empresa, el año y el carácter exportador.

Posteriormente se realiza un análisis de la varianza para descubrir cuáles son los factores que provocan cambios en el valor de la rentabilidad económica. Se evalúa si el efecto del sector económico y la localización de la empresa influyen significativamente en la respuesta (rentabilidad). Como última etapa, se utiliza una regresión lineal múltiple para averiguar en qué medida el sector de actividad, número de empleados, facturación, localización, exportación, tiempo y $\mathrm{PIB}^{2}$.

\footnotetext{
${ }^{1}$ Incluye la evolución de más de 10.000 empresas durante el período de referencia.

${ }^{2}$ Datos obtenidos a partir del Instituto Nacional de Estadística (INE).
} 


\section{RESULTADOS}

Las provincias de A Coruña y Pontevedra son las que cuentan con más empresas, independientemente del sector, dentro de la Comunidad Autónoma de Galicia. Los sectores más representativos son la agricultura, construcción, detallistas, fabricantes, mayoristas y servicios. El porcentaje de empresas exportadoras está en torno al 10\% del total de pymes estudiadas. A partir del Gráfico $\mathrm{n}^{\circ} 1 \mathrm{y} \mathrm{n}^{\circ} 2$ se puede observar el número de pymes exportadoras a nivel sectorial, siendo mayor en el sector de fabricantes y mayoristas.

Para este análisis, se parte de una base de datos reducida. Se han eliminado aquellas observaciones de la rentabilidad detectadas como atípicos, es decir, mayores que Q3+1.5(Q3-Q1) y menores que Q11.5(Q3-Q1). La inclusión de empresas con rentabilidades extremadamente altas o bajas impediría, para las empresas más características de la población, analizar convenientemente la estructura de dependencia de esta variable con respecto a las demás.

A partir de los histogramas de las rentabilidades de las pymes gallegas para cada año, exportadoras o no, se observa el efecto de la crisis a partir de 2008. La cola derecha de los histogramas, que caracteriza a la proporción de compañías con una rentabilidad elevada, es cada vez menor. Sin embargo, el área bajo la cola izquierda se incrementa. El eje de simetría de los histogramas se desplaza, con independencia de su carácter exportador, hacia rentabilidades menores.

Si comparamos las funciones de densidad, estimadas mediante el método kernel gausiano y ventana óptima obtenida por el método de Seather y Jones (Venables y Ripley, 2002), se observan muy ligeras diferencias entre las empresas que exportan y las que no lo hacen. La probabilidad de que la empresa tenga una rentabilidad media, entre cuatro y cinco, es ligeramente superior en las empresas exportadoras. 


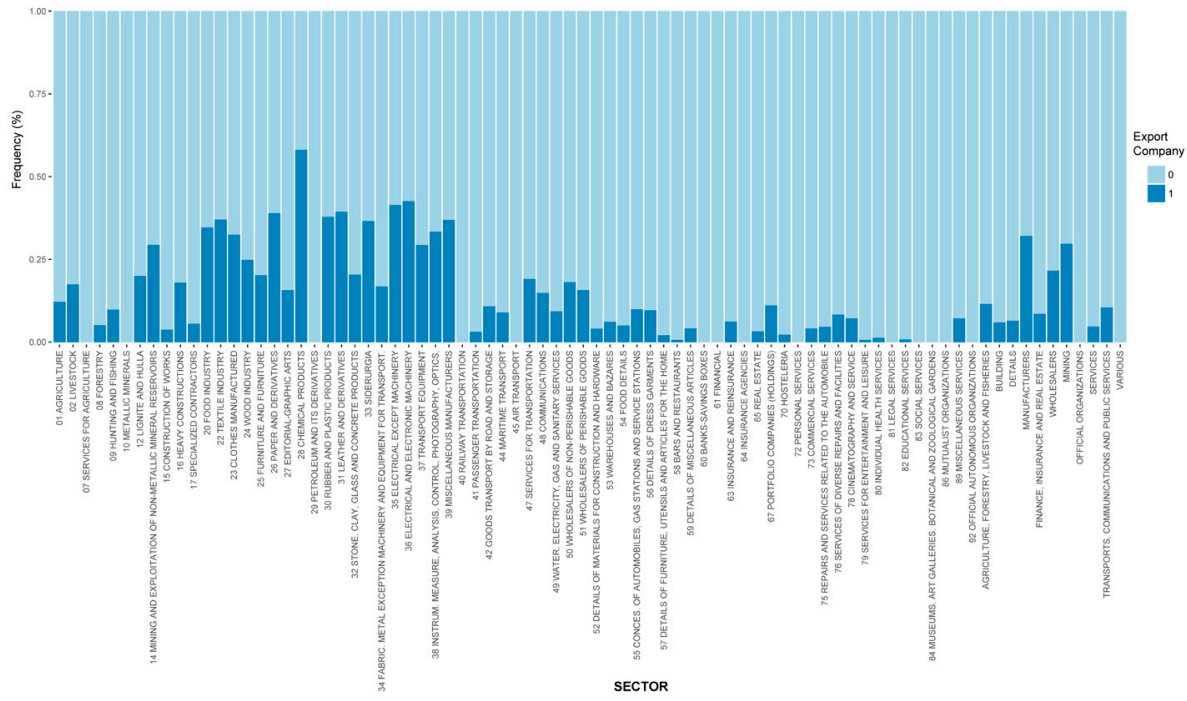

Gráfico 1. Pymes exportadoras por sector.

Fuente: elaboración propia a partir de ARDAN.

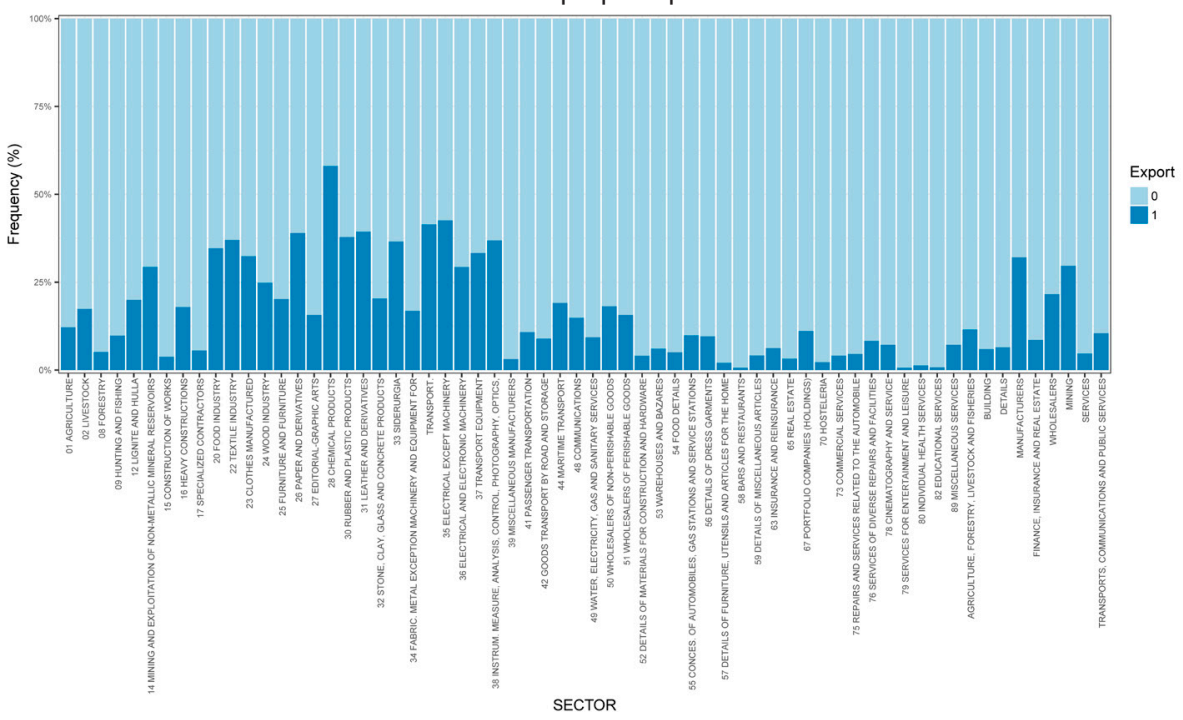

Gráfico 2. Sectores con Pymes exportadoras.

Fuente: elaboración propia a partir de ARDAN. 
Así, con anterioridad a la crisis, las compañías que exportaban tenían una mayor rentabilidad media. Durante los primeros años de la crisis, y hasta el año 2011, se ha observado que la rentabilidad de las empresas exportadoras fue ligeramente menor. La rentabilidad cayó desde seis a menos de 3,5 durante los peores años de la crisis. Ese año la rentabilidad de ambos tipos de empresas converge. Con posterioridad a 2012 se observa una tendencia ascendente, donde las empresas exportadoras presentan una rentabilidad media más elevada, aunque siempre teniendo en cuenta que las diferencias son muy escasas.

A partir de lo comentado anteriormente, el hecho de que una empresa exporte o no, podría afectar, aunque solo fuera muy ligeramente, a su rentabilidad. Igualmente, puede decirse del año (efecto de la crisis). Así, nos surge la pregunta ¿qué factores pueden provocar cambios significativos en el valor de la rentabilidad?

Todos los factores, salvo la exportación, influyen significativamente en el valor de la rentabilidad.

A partir de la aplicación del test $\mathrm{F}$, donde la hipótesis nula que se contrasta es que la media de la rentabilidad es igual para cualquier nivel de año, exportación, localización o sector (utilizado un p-valor de 0.05), se concluye que todos los factores, salvo la exportación, influyen significativamente en el valor de la rentabilidad. Sin embargo, si en lugar de estudiar los efectos principales, se estudian también las interacciones entre factores, se obtiene un resultado complementario indicando que el hecho de exportar si influye en el valor de la rentabilidad.

La interacción entre año y exportación provoca cambios significativos en la rentabilidad y es diferente según el año. Antes de la crisis el efecto de la exportación se traducía en un incremento de la rentabilidad. Durante los primeros años de la crisis afectaba negativamente a la rentabilidad y, posteriormente, positivamente de nuevo.

El modelo obtenido del análisis mediante la regresión líneal múltiple fue el siguiente:

$$
\begin{gathered}
\text { ROA }=\text { Sector }+ \text { Exportación }+1,61 * 10^{-3} \text { Empleo }+1,04 * 10^{-8} \text { Facturación }+ \text { Localización }- \\
0,21 \text { Tiempo }+3,28 * 10^{-6} \text { PIB }
\end{gathered}
$$

Se obtuvo como resultado de esta modelización que la relación entre la rentabilidad y el resto de variables es muy débil. Aunque se comprueba que existe una dependencia estadísticamente 
significativa, el modelo lineal resultante no proporcionaría estimaciones fiables de la rentabilidad para una nueva empresa.

Aumentos en el PIB, el número de empleados y la facturación se relacionan con incrementos en la rentabilidad, aunque la evolución en el tiempo se corresponde con un decremento en la misma.

Se observa que aumentos en el PIB, el número de empleados y la facturación están relacionados con incrementos en la rentabilidad. Por el contrario, la evolución en el tiempo se corresponde con un decremento en la rentabilidad. 


\section{CONCLUSIONES}

El primer hecho relevante que se desprende de este trabajo es que las empresas que exportan representan un porcentaje muy pequeño de la muestra, una décima parte. Así, el grado de internacionalización empresarial de la región estudiada es escaso. Además, también es destacable que las rentabilidades que presentan las empresas exportadoras son muy similares a las que no presentan ninguna actividad internacional.

Se observa una conducta ligada al ciclo económico y a la influencia del tiempo. Las firmas que ostentaban un enfoque internacional antes de la crisis presentaban una mayor rentabilidad media.

La influencia de la exportación sobre la rentabilidad revela la necesidad de ahondar en las consecuencias que tiene la primera sobre la gestión empresarial.

El análisis ha puesto de manifiesto qué, a excepción de la exportación, el resto de factores incluidos para explicar la rentabilidad empresarial influyen en ella significativamente. Sin embargo, si observamos las interacciones entre los factores, la exportación sí que influye sobre la rentabilidad. Este hecho revela la necesidad de ahondar en las consecuencias que tiene la exportación sobre la gestión empresarial.

La modelización propuesta, a través de una regresión lineal, no ha obtenido estimaciones fiables. Esto inicia el camino tanto a la utilización de nuevas variables explicativas como a utilizar otro tipo de modelos (por ejemplo, SVM o redes neuronales). El estudio de la propensión a exportar es otra cuestión ampliamente tratada en la literatura y una evolución natural de este trabajo. De este modo, se abre la posibilidad de emplear modelos logit o similares.

Asimismo, después de observar la distribución sectorial de las empresas exportadoras, se muestra interesante analizar estos sectores específicos. Al mismo tiempo, y dada la distribución espacial de las empresas a lo largo del territorio, enfocarse a subniveles geográficos específicos también es un ámbito relevante para ser explorado. 


\section{REFERENCIAS BIBLIOGRÁFICAS}

Annavarjula, M., y Beldona, S. (2000). Multinationality-performance relationship: a review and reconceptualization. International Fournal of Organizational Analysis, 8(1), 48-67.

ARDAN. (Varios años). Base de datos ARDAN. Vigo: Consorcio de la Zona Franca de Vigo. Departamento de servicios avanzados - ARDAN.

Bernard, A. B., y Jensen, J. B. (1999). Exceptional exporter performance: Cause, effect, or both? Journal of International Economics, 47(1), 1-25.

Boehe, D. M., Qian, G., y Peng, M.W. (2016). Export intensity, scope and destinations: Evidence from Brazil. Industrial Marketing Management, 57, 127-138.

Contractor, F. J., Kundu, S.K., y Hsu, G-C. (2003). A three-stage theory of international expansion: the link between multinationality and performance in the service sector. Fournal of International Business Studies, 34, 5-18.

Ellis, P.D., Davies, H., y Wong, A. H-K. (2011). Export intensity and marketing in transition economies: Evidence from China. Industrial Marketing Management, 40(4), 593-602.

Giovannetti, G., Ricchiuti, G. y Velucchi, M. (2013). Location, internationalization and performance of firms in Italy: a multilevel approach. Applied Economics, 45, 2665-2673.

Glaum, M., y Oesterle, M.J. (2007). 40 years of research on internationalization and firm performance: More questions than answers? Management International Review, 47(3), 307-317.

Gomes, L., y Ramaswamy, K. (1999). An empirical examination of the form of the relationship between multinationality and performance. Fournal of International Business Studies, 30, 173188.

Gourlay, A., y Seaton, J. (2004). Explaining the decision to export: evidence from UK firms. Applied Economics Letters, 11, 153-158.

INE. (Varios años). Contabilidad Nacional anual de España. Madrid: Instituto Nacional de Estadística. Recuperado de: http://www.ine.es/dyngs/INEbase/es/operacion. htm?c=Estadistica_C\&cid $=1254736165950 \&$ menu $=u l t i D a t o s \& i d p=1254735576581$ 
Javalgi, R., White, D.S. y Lee, O. (2000). Firm characteristics influencing export propensity: An empirical investigation by industry type. Fournal of Business Research, 47, 217-228.

Johanson, J. y Vahlne, J.E. (1977). The internationalization process of the firm - a model of knowledge development and increasing foreign market commitment. Fournal of International Business Studies, 8(1), 23-32.

Kalinic, I., y Forza, C. (2012). Rapid internationalization of traditional SMEs: Between gradualist models and born globals. International Business Review, 21(4), 694-707.

Kamakura, W.A., Ramón-Jerónimo, M.A., y Vecino Gravel, J.D. (2012). A dynamic perspective to the internationalization of small-medium enterprises. Fournal of the Academy Marketing Science, 40, 236-251.

Li, L. (2007). Multinationality and performance: A synthetic review and research agenda. International Journal of Management Reviews, 9(2), 117-139.

Li, L., y Qian, G. (2005). Dimensions of international diversification: the joint effects on firm performance. Fournal of Global Marketing, 18(3/4), 7-35.

Ling-Yee, L. (2004). An examination of the foreign market knowledge of exporting firms based in the People's Republic of China: Its determinants and effect on export intensity. Industrial Marketing Management fournal, 22(6/7), 656-586.

Lu, J.W., y Beamish, P.W. (2004). International diversification and firm performance: the S-curve hypothesis. Academy of Management fournal, 47, 598-609.

Majocchi, A., Dalla Valle, L., y D’Angelo, A. (2015). Internationalisation, cultural distance and country characteristics: A bayesian analysis of SMEs financial performance. Fournal of Business Economics and Management, 16(2), 307-324.

Oviatt, B.M. y McDougall, P.P. (1994). Towards a theory of international new ventures. Fournal of International Business Studies, 25(1), 45-64.

Pangakar, N. (2008). Internationalization and performance of small-and medium-sized enterprises. Journal of World Business, 43, 475-485. 
Pantzalis, C. (2001). Does location matter? An empirical analysis of geographic scope and MNC market evaluation. Fournal of International Business Studies, 32, 133-155.

Ramírez-Aleson, M., y Espitia-Escuer, M.A. (2001). The effect of international diversification on performance. Management International Review, 41, 291-315.

Serra, F., Pointon, J., y Abdou, H. (2012). Factors influencing the propensity to export: a study of UK and Portuguese textile firms. International Business Review, 21, 210-224.

Serti, F., y Tomasi, Gh. (2014). Export and import market-specific characteristics. Empirical Economics, 47, 1467- 1496.

Venables, W. N., y Ripley B. D. (2002). Modern Applied Statistics with S. Springer.

Westhead, P., Wright, M., y Ucbasaran, D. (2001). The internationalization of new and small firms: A resource-based view. Fournal of Business Venturing, 16(4), 333-358.

Yi, J. y Wang Ch. (2012). The decision to export: Firm heterogeneity, sunk costs, and spatial concentration The decision to export: Firm heterogeneity, sunk costs, and spatial concentration. International Business Review, 21, 766-781. 


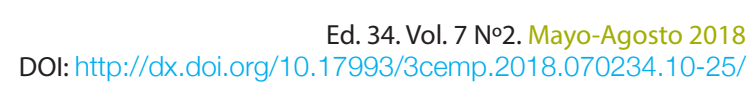

